DOI: https://doi.org/10.46296/rc.v5i9.0046

\title{
Potencialidades de inclusión de Pleurotus ostreatus en alimentos cárnicos
}

\section{Potentialities of inclusion of Pleurotus ostreatus in meat foods}

\author{
Álvarez-Serrano Isaris María \\ Profesora del departamento de Ciencia y Tecnología de los Alimentos, \\ Universidad de Camagüey Ignacio Agramonte Loynaz. Camagüey, Cuba. \\ Correo: isaris.alvarez@reduc.edu.cu \\ ORCID ID: https://orcid.org/0000-0001-7772-6262

\section{Mustelier-Casola Daimara} \\ Profesora del departamento de Ciencia y Tecnología de los Alimentos, \\ Universidad de Camagüey Ignacio Agramonte Loynaz. Camagüey, Cuba. \\ Correo: daimara.mustelier@reduc.edu.cu \\ ORCID ID: http://orcid.org/0000-0001-5284-5046 \\ González-Zambrano José Miguel \\ Ingeniero Agrícola. Tecnólogo Agrícola. Manabí, Ecuador. \\ Correo: miguellgonzalezz9@gmail.com \\ ORCID ID: https://orcid.org/0000-0002-9567-3309
}

\begin{abstract}
Espinosa-Nieto Leticia
Profesora Auxiliar del departamento de Ciencia y Tecnología de los Alimentos, Universidad de Camagüey Ignacio Agramonte Loynaz. Camagüey, Cuba. Correo: leticia.espinosa@reduc.edu.cu ORCID ID: http://orcid.org/0000-0002-4181-4276
\end{abstract}

\begin{abstract}
RESUMEN
Las setas comestibles como Pleurotus ostreatus resultan una buena opción para formulaciones cárnicas, dado su contenido de nutrientes; además, posee compuestos bioactivos que le confieren propiedades antioxidantes, antimicrobianas y anticancerígenas, principalmente. El presente trabajo valora las potencialidades del uso de Pleurotus ostreatus en productos cárnicos. El diseño de la investigación fue no experimental. El estudio sostuvo como método la observación no participativa para la recopilación de información, y como técnica al análisis documental para la obtención de datos reales y el fortalecimiento de resultados. Se afirma que la adición de hongos comestibles, específicamente de Pleurotus ostreatus en productos cárnicos mejora la calidad nutricional de estos productos, ha sido empleado de manera satisfactoria en varias formulaciones de alimentos como hamburguesas de pollo, de res, salchichas de cerdo y tipo vienesa, paté de hígado y Nuggets de pollo. Además, el cultivo del hongo en sustratos como mazorcas de cacao, orujos de pera, briquetas de bagazo de caña de azúcar ha demostrado sostenibilidad, al posibilitar resolver problemas medioambientales. En Camagüey se potencia el uso de la cascarilla de arroz dada su disponibilidad, bajo precio, cercanía de las plantas generadoras y contribución a minimizar el impacto ambiental.
\end{abstract}

Palabras claves: Pleurotus ostreatus, cárnicos, nutrición, sustrato.

Información del manuscrito:

Fecha de recepción: 01 de diciembre de 2021.

Fecha de aceptación: 27 de diciembre de 2021.

Fecha de publicación: 06 de enero de 2022. 


\begin{abstract}
Edible mushrooms such as Pleurotus ostreatus are considered a good option as an ingredient in the formulations of meat products, due to their high content of nutrients, the bioactive compounds present in their composition give them antioxidant, antimicrobial, anticancer properties, among others. The present work values the potentialities of the use of Pleurotus ostreatus in meat products. The research design was non-experimental. The study also supported non-participatory observation as a method for the collection of information, and as a technique, documentary analysis to obtain real data and strengthen the results. It could be stated that the addition of edible mushrooms, specifically Pleurotus ostreatus in meat products can improve the nutritional quality of these products. Well, it has been used successfully in various food formulations such as chicken burgers, beef burgers, pork sausage, Viennese type sausage, liver pate, and chicken nuggets. In addition, the cultivation of the fungus on different substrates such as cocoa pods, pear marc, and sugarcane bagasse briquettes has demonstrated its sustainability by allowing it to solve environmental problems. In Camagüey, studies were carried out in this regard, highlighting the rice husk for its great availability, low price and proximity to the generating plants, helping to minimize its impact on the environment.
\end{abstract}

Keywords: Pleurotus ostreatus, meat foods, nutrition, substrate.

\title{
1. INTRODUCCIÓN
}

La carne y sus derivados, de acuerdo a su valor nutricional se caracterizan por su significativo aporte proteico, su alto contenido de grasa, principalmente grasa saturada. Además, tienen un elevado contenido de vitaminas y minerales. No obstante, debido a su alta cantidad de grasa saturada, sal y nulo aporte de fibra y unido también al estilo de vida de cada individuo, su consumo está asociado a factores de alto riesgo para el padecimiento de enfermedades crónicas. Dentro de las cuales figuran enfermedades de origen cardiovascular, síndrome metabólico, diabetes y algunos tipos de cáncer como el cáncer de colon (Cerón et al., 2020). Por tanto, optar por el consumo de alimentos más saludables, ligado a la adición y/o reemplazo de ingredientes naturales en formulaciones cárnicas conllevaría al mejoramiento de la calidad nutricional de los productos cárnicos y así se podría reducir el riesgo de distintos padecimientos.

En ese sentido, se han llevado a cabo diversas investigaciones enfocadas en crear variantes encaminadas a la utilización de fuentes naturales. Han sido estudiadas proteínas y fibras dietéticas de subproductos de vegetales, legumbres, cereales, tubérculos, semillas comestibles, hasta de fuentes poco utilizadas como algas o insectos, como fuente de ingrediente o aditivo para 
mejorar las propiedades nutricionales y funcionales de los alimentos. Los hongos comestibles son una de las fuentes recientemente redescubiertas de varios ingredientes funcionales. Debido a que son conocidos desde la antigüedad y eran considerados por los romanos como "alimento de los dioses". Además, son muy explotados por los países asiáticos gracias a sus propiedades nutracéuticas y terapéuticas (Rangel et al., 2021).

Es por ello que las setas comestibles como Pleurotus ostreatus son consideradas una buena opción como ingrediente en las formulaciones de productos cárnicos, debido a que presentan compuestos bioactivos, propiedades antioxidantes, antimicrobianas, anticancerígenas entre otras. Entre estos compuestos, se encuentran los $\beta$-glucanos, que han mostrado múltiples beneficios para la salud humana (Cerón et al., 2020), (Wan Rosli y Solihah, 2012). Esto ha atraído la atención hacia el uso de hongos como posibles sustitutos de varios ingredientes y aditivos en alimentos procesados, principalmente productos cárnicos (Rangel et al., 2021).

Además, de los beneficios nutricionales atribuidos a las setas comestibles, también existe un elemento muy importante que es la disponibilidad de sustratos potenciales para su cultivo, que son de fácil adquisición y permiten resolver problemas medioambientales (Puig, Crespo, Cardona, Matos y Serrano, 2020) lo que permite una producción sostenible de este tipo de hongo e incluso podría contribuir a la sustitución de importaciones.

En el mundo se han realizado múltiples estudios con relación a este tema y Cuba no ha quedado exenta de ello. Se han desarrollado investigaciones análogas en diferentes instituciones. No obstante, no se ha logrado fomentar una industria productora de setas comestibles con posibilidades de introducción en los diferentes grupos de alimentos, lo que permite la posibilidad de desarrollar alimentos funcionales a base de setas comestibles. Es por ello que esta investigación tuvo como objetivo: valorar las potencialidades de Pleurotus ostreatus para su utilización en productos cárnicos. 


\section{MATERIALES Y MÉTODOS}

El presente estudio, se realizó mediante una revisión bibliográfica utilizando fuentes de información primarias y secundarias, lo que posibilitó la confección del marco de referencia teórico y metodológico. El diseño de la investigación fue no experimental, transaccional y correlacional. El estudio sostuvo además como método a la observación no participativa para la recopilación de información, y como técnica al análisis documental para la obtención de datos reales y el fortalecimiento de los resultados. En la metodología que se utilizó se consideraron elementos importantes como las propiedades físicas y microbiológicas de Pleurotus ostreatus, los beneficios que trae para la salud humana el consumo de setas comestibles y la disponibilidad de sustratos para su cultivo. Por tanto, se enfatizó en su utilización en alimentos cárnicos.

\section{RESULTADOS}

\subsection{Aspectos nutricionales y benéficos de los hongos comestibles}

Los hongos comestibles tienen un alto contenido de agua (82 \% - $92 \%$ ), son bajos en calorías, según De la Cruz (2020). Poseen una baja cantidad de grasa (2 \% a $6 \%$ en base seca). El nivel de ácidos grasos poliinsaturados en comparación con los saturados es alto, constituyendo más del 75 \% de los ácidos grasos totales, de los cuales resaltan el ácido palmítico, ácido oleico y linoleico. Respecto al contenido de minerales destacan el potasio, fósforo, hierro, zinc, magnesio, selenio, sodio y calcio. Son fuentes importantes de proteínas, en comparación con la mayoría de las verduras, contienen en peso seco entre 10 \% a $30 \%$ de proteína. El contenido de aminoácidos oscila entre $34 \%$ a $47 \%$, destacándose treonina, valina, lisina, leucina, isoleucina y triptófano. Son también fuente de vitaminas hidrosolubles y liposolubles como tiamina, riboflavina, pirodoxina, cianocobalamina, ácido pantoténico, niacina, ácido fólico, vitamina $A$, vitamina $D$, vitamina $E$ y vitamina $K$. Son ricos en carbohidratos (35 $\%$ a $70 \%$ en peso fresco). Contienen un alto nivel de oligosacáridos, los polisacáridos presentes son quitina, manitol y trehalosa. Por otra parte, la fibra dietética del hongo es la parte comestible que es resistente a la digestión y absorción en el intestino delgado humano. Su presencia varía entre el 27 \% y el 
$44 \%$ en base seca, comprende quitina, hemicelulosa, mananos y ßglucanos (contienen de 4 a $13 \%$ de $\beta$ glucanos, presentes como fibra dietética total). Estos compuestos bioactivos, unidos a polisacáridos, proteínas, compuestos fenólicos (flavonoides, lignanos y ácidos fenólicos), ligninas, triterpenos han mostrado múltiples beneficios para la salud humana debido a que presentan propiedades antioxidantes, antimicrobianas, anticancerígenas inmunoreguladora, antiglicémica y hepatoprotectora, entre otras. Es por ello que se recomienda como un alimento ideal (Rangel et al., 2021), (Cerón et al., 2020), (De la Cruz et al., 2020), (González, 2016), (Ruilova et al., 2014), (Wan Rosli, 2011) y (Wan Rosli, y Solihah, 2012).

\subsection{Sustratos y suplementos nutricionales empleados en el crecimiento de Pleurotus sp}

Existen múltiples reportes de sustratos y suplementos nutricionales empleados en el crecimiento de Pleurotus sp. como pajas, rastrojos, pulpas, bagazos, residuos forestales (Piña, 2016).

En un estudio realizado por Hurtado, Huamán, Bravo, Silva y Silva (2016) se evaluó el cultivo de Pleurotus ostreatus en mazorcas de cacao (Theobroma cacao L.). En este estudio se describió el cultivo y producción de Pleurotus ostreatus utilizando mazorcas de cacao como sustrato. El cultivo de P. ostreatus se realizó en ocho etapas, donde se evaluaron el efecto del tiempo de fermentación (1, 3 y 7 días) y el tratamiento térmico del sustrato (pasteurización y esterilización) sobre la producción de P. ostreatus. Se evaluó el peso de carpóforos, la eficiencia biológica (E.B) y el rendimiento (R), en seis tratamientos con cinco repeticiones. Para el experimento se utilizó un diseño completamente al azar (DCA) mediante un análisis de varianza (ANOVA). Además, se realizó la prueba de rangos múltiples de Tukey a los tratamientos. El tratamiento que resultó significativamente diferente y con mejores resultados en comparación con los demás fue el tratamiento con 3 días de fermentación tratado por esterilización (T5), que obtuvo una producción de $283 \mathrm{~g}$ de hongos recolectados en dos cosechas, $31,22 \%$ de eficiencia biológica y $28,30 \%$ de rendimiento.

Rodríguez, Martínez, Buglione, Filippi y Agüero (2018), evaluaron la factibilidad de emplear orujo de pera como sustrato para el cultivo comercial de dos cepas 
de Pleurotus ostreatus (PI-P y PI-J) y analizaron la variación de la composición química del orujo biodegradado. En este caso la cepa PI-J presentó $98,3 \%$ de Eficiencia Biológica, $35 \%$ de Rendimiento y 45 días de Período Productivo, mientras que para PI-P los valores fueron $62,3 \%, 21,8 \%$ y 35 días, respectivamente. La actividad metabólica de ambas cepas sobre el sustrato provocó una disminución relativa del contenido de materia seca y materia orgánica del $20 \%$ al $30 \%$ y de un $60 \%$ para los carbohidratos solubles $(p<$ 0,05). Al finalizar el Período Productivo, las fibras (FDN, FDA y LDA) disminuyeron $10 \%$ a $20 \%$ mientras que el contenido de proteína bruta no se modificó significativamente.

En otro estudio se evaluó el bagazo de Agave salmiana ssp. crassispina y Agave angustifolia ssp. tequilana var. azul, subproductos de la industria del mezcal en diferentes tratamientos con salvado de trigo, viruta de pino, viruta de nogal, viruta de cedro y paja de avena, usado como sustrato para el cultivo de Pleurotus ostreatus. Se realizó análisis bromatológico a cada tratamiento al inicio del cultivo, determinando las variables de humedad, cenizas, fibra cruda, proteína cruda, lípidos, azúcares reductores totales; así como cuantificación de la eficiencia biológica de P. ostreatus. Se analizó estadísticamente mediante la prueba HSD de Tukey, registrando que la mezcla bagazo de A. angustifolia ssp. tequilana var. azul mezclado con $30 \%$ de viruta de nogal y $5 \%$ de salvado de trigo, obtuvo la eficiencia biológica de 33,24 \% (Heredia et al., 2016).

En un estudio realizado por Magalhães, Moreira y Zied (2018) se evaluó el rendimiento productivo de $\mathrm{P}$. ostreatus cultivado en briquetas de bagazo de caña de azúcar suplementadas con bioproductos de cereales. En 30 días de cultivo axénico, las briquetas de 6 toneladas de fuerza con $80 \%$ de humedad resultaron en una mayor productividad $(30,4 \%)$, y generaron el menor volumen físico de compuesto agotado (44,8\%). Por tanto, el cultivo axénico de P. ostreatus var. Florida en briquetas de bagazo de caña de azúcar, complementado con bioproductos de cereales, se ha calificado técnicamente como un método original y eficiente para la producción de proteína de alto valor biológico mediante el reciclaje de residuos agrícolas y por lo tanto puede garantizar múltiples beneficios económicos a la agroindustria de P. ostreatus, además de permitir la 
reducción de impactos ambientales por la generación restringida de compuesto agotado en la etapa de poscosecha.

Los sustratos que se empleen dependen en gran medida de las disponibilidades agrícolas de cada región. En Cuba se han empleado fundamentalmente para el cultivo sustratos como bagazo de caña de azúcar, pulpa de café y cáscara de cacao (García, Bermúdez, Serrano, 2011). En un estudio realizado en la provincia Camagüey (Puig, Crespo, Cardona, Matos y Serrano, 2020) se evaluó la cascarilla de arroz y el afrecho cervecero como sustratos puros para el cultivo del Pleurotus ostreatus, empleando la pulpa de café como referencia. Se utilizó la cepa CCEBI 3024 Pleurotus ostreatus var. Florida. Los parámetros estudiados fueron: eficiencia biológica, rendimiento, diámetro de los cuerpos fructíferos y precocidad. El estudio reveló que el afrecho cervecero no fue un medio propicio para la fructificación de la seta objeto de estudio en las condiciones de producción. El sustrato de referencia pulpa de café superó en cuanto a eficiencia, precocidad y rendimiento a la cascarilla de arroz con respectivos valores de eficiencia biológica y rendimiento de 68,02 \% y 17,00 \% sobre la pulpa de café y $33,03 \%$ y $15,19 \%$ en cascarilla de arroz. No obstante, este último posee potencialidades para ser empleado en el cultivo de setas comestibles en la localidad de Camagüey por su gran disponibilidad, bajo precio y cercanía de las plantas generadoras, contribuyendo a minimizar su impacto en el medio ambiente. Lo cual demuestra la disponibilidad que existe de sustratos potenciales para su cultivo, que son de fácil adquisición y permiten resolver problemas medioambientales, lo que permite una producción sostenible de este tipo de hongo.

\subsection{Análisis sobre las posibilidades de inclusión de setas comestibles en alimentos cárnicos}

La inclusión de setas comestibles de Pleurotus osteatrus en alimentos cárnicos ha sido objeto de estudio en la industria alimentaria cubana, tomando como fundamentos investigaciones de Wan Rosli (2011), en que se elaboraron hamburguesas de pollo, formuladas con $0 \%, 25 \%$ o $50 \%$ de setas de Pleurotus fresco. Los resultados muestran que la hamburguesa de pollo formulada con un $25 \%$ de la seta tenía un contenido proteico de $17.46 \%$ menor que la 
hamburguesa de control que tenía $18.13 \%$, lo cual no fue significativo. Sin embargo, el contenido proteico se reduce significativamente cuando la carne de pollo se reemplaza con un $50 \%$ de la seta. Las hamburguesas de pollo que contienen $25 \%$ y $50 \%$ de PSC registraron significativamente una menor concentración de grasa al 10,67\% y 7,15 \%, respectivamente. Además, la hamburguesa incorporada con un $25 \%$ tenía un contenido de humedad del 56,91 $\%$, que es significativamente menor que la hamburguesa formulada con $50 \%$ que tenía una humedad del 58,80 \%. En la evaluación sensorial, no se registraron diferencias en los atributos sensoriales. La incorporación de la seta molida para reemplazar parcialmente la carne de pollo reduce la grasa y las cenizas, pero aumenta el contenido de humedad de las hamburguesas cocidas. Los autores recomiendan la adición de la seta al $25 \%$ para reemplazar parcialmente la carne de pollo para reducir el costo de producción y el contenido de grasa sin cambiar el contenido de proteína, tomando en consideración que el contenido de proteína disminuyó.

Estos autores realizaron un estudio similar pero con hamburguesas de carne de res. En este caso se investigó la capacidad del hongo para cambiar las características físicas y las propiedades sensoriales de la carne de res formulada con este hongo. El resultado muestra que la hamburguesa de res añadida con un 50 \% de la seta molida registró la concentración más alta de fibra dietética total a 9,95 g / $100 \mathrm{~g}$ en comparación con la hamburguesa de res que contiene un $25 \%$ de la seta $(7,00 \mathrm{~g} / 100 \mathrm{~g})$. La carne de res, que fue reemplazada con un $25 \%$ del hongo, registró el mayor rendimiento de cocción (76,62 \%) y retención de humedad (59,80 \%), respectivamente. En los otros rasgos físicos, la hamburguesa de res que contenía un $25 \%$ del hongo registró una retención de grasa del $89,04 \%$ y no fue significativa $(p<0,05)$ con la hamburguesa de control $(88,59 \%)$. Por tanto, los autores recomiendan la adición del hongo al 25 $\%$, lo cual ayuda a la reducción del costo de la formulación sin afectar los descriptores sensoriales del producto con los que el consumidor está familiarizado (Wan Rosli y Solihah, 2012).

Se reporta en la literatura la elaboración de hamburguesas a partir de setas frescas y de setas deshidratadas, en los que se encontró que ambos productos contenían niveles de proteínas entre 38,26 \% y 35,23 \%; de grasa entre 13,91 \% 
y $8,34 \%$ y de carbohidratos entre $17,52 \%$ y 14,92 \%. El sustituto cárnico elaborado con las setas deshidratadas tuvo mayor objeción sensorial debido a la textura (Jaramillo et al., 2011).

En otra investigación se desarrolló un sustituto más saludable de la salchicha de cerdo fermentada tailandesa; un producto popular tradicional que, sin embargo, contiene niveles muy altos de grasas saturadas. Los hongos se utilizaron como sustituto de la carne de cerdo. El producto final se caracterizó mediante análisis microbiológico y proximal y se llevó a cabo una evaluación sensorial. En conclusión, la producción de una alternativa a la salchicha tailandesa fermentada tradicional es posible utilizando P. ostreatus más económico y saludable. El producto final, que contiene una proporción de setas a arroz glutinoso de 40:60, fue aceptable para los panelistas y se comparó favorablemente con las salchichas fermentadas tradicionales en términos de valores caloríficos y estándares microbianos (Chockchaisa Wasdee, 2010).

Ruilova, Hernández, Díaz y Niño-Ruiz (2014) estudiaron una formulación óptima (40\% de carne de res; $27 \%$ de hongo y $8 \%$ de grasa) para la salchicha tipo vienesa cambiando el contenido de carne de cerdo por el hongo. Los resultados obtenidos de la evaluación de la emulsión carne-hongo-grasa de las formulaciones experimentales, apoyan el criterio del empleo del hongo en productos de pasta fina. Los resultados de las propiedades funcionales del hongo y de la emulsión carne hongo permitieron confirmar sus características beneficiosas y adecuadas para su utilización como materia prima en sustitución de la carne en productos cárnicos convencionales. Fue posible optimizar una formulación baja en grasa y nitritos, utilizando al Pleurotus ostreatus como sustituto de la carne de cerdo, la adición del hongo no afectó mayormente la composición nutricional del nuevo producto, si no por el contrario presentó características beneficiosas en su composición por la presencia de $\beta$-glucanos y fibra.

Se reportan también estudios en que se elaboran croquetas, que además tras su elaboración industrial o casera deben someterse a una fritura doméstica. Tanto el proceso de elaboración como la fritura doméstica pueden modificar las propiedades fisicoquímicas del producto y, por lo tanto, pueden afectar también 
la capacidad antioxidante del producto, es por ello que se prefiere la elaboración de paté y se han reportado estudios al respecto (Ramírez 2009). El paté de hígado de cerdo es una pasta untable que se comercializó como sustituto más económico del típico "foie-grass" francés, obtenido a partir del hígado de cierto tipo de gansos. Los patés de hígado de cerdo presentan un alto valor nutricional debido a su elevado contenido en hierro, aunque hay que destacar que también presentan un elevado contenido graso.

Setas de Pleurotus molidas secas se emplearon en la elaboración de nuggets de pollo. Los autores, recomendaron un $10 \%$ de sustitución de la carne por harina de hongos para evitar una dureza excesiva, un color oscuro y un sabor desagradable derivado de concentraciones más altas de hongos (Wan -Mohtar et al., 2020).

En Cuba, se han desarrollado investigaciones similares en instituciones como el Instituto de Investigaciones de la Industria Alimentaria, en el Instituto de Farmacia y Alimentos, y algunos centros de las FAR y el MININT. No obstante, hasta el momento no ha sido posible fomentar una industria productora de setas comestibles con posibilidades de introducción en los diferentes grupos de alimentos, lo que permite la posibilidad de desarrollar alimentos funcionales a base de setas comestibles.

El territorio camagüeyano cuenta con industrias productoras de alimentos cárnicos tales como TERSO y la empresa cárnica de Camagüey, que constituyen entidades ejecutoras participantes en el proyecto "Desarrollo de tecnologías para la producción de alimentos a partir de setas comestibles en la cadena agroalimentaria", y existe la disposición y el compromiso, por parte de las direcciones administrativas y técnicas, de suministrar las materias primas necesarias para la elaboración de los productos y de utilizar las instalaciones industriales para la elaboración. También se prevé la posibilidad, a largo plazo, de obtener las sepas en las propias industrias con el objetivo de autoabastecerse y de garantizar la sostenibilidad de sus producciones.

Hasta el momento de ejecución del proyecto "Desarrollo de tecnologías para la producción de alimentos a partir de setas comestibles en la cadena agroalimentaria" se ha constatado con las direcciones de las dos empresas. Se 
han realizado reuniones de trabajo en que se ha expuesto, por parte de los investigadores del proyecto, las potencialidades nutricionales y de alimentos saludables que poseen los productos cárnicos con un determinado porcentaje de inclusión de setas comestibles. En ambas industrias se ha destinado parte del personal técnico para la capacitación, atendiendo a la adaptabilidad de la tecnología instalada para la utilización de esta materia prima.

\section{CONCLUSIONES}

Los hongos comestibles se consideran una excelente alternativa como ingrediente en las formulaciones de productos cárnicos por su bajo contenido calórico y de grasa. Presentan también, compuestos bioactivos, con propiedades antioxidantes, antimicrobianas, anticancerígenas entre otras. Entre estos compuestos, se encuentran los $\beta$-glucanos, que han mostrado múltiples beneficios para la salud humana. De esta manera, se puede afirmar que la adición de hongos comestibles, específicamente de Pleurotus ostreatus en productos cárnicos puede mejorar la calidad nutricional de estos productos. Se ha empleado en varias formulaciones de alimentos como en hamburguesas de pollo, de res, salchicha de cerdo, salchicha tipo vienesa, en paté de hígado, en Nuggets de pollo. Además, el cultivo del hongo en diferentes sustratos (mazorcas de cacao, orujos de pera, briquetas de bagazo de caña de azúcar) ha demostrado su sostenibilidad al permitir resolver problemas medioambientales. Estudios realizados en la Universidad de Camagüey, potencian la cascarilla de arroz como sustrato, dada su disponibilidad, bajo precio y cercanía de las plantas generadoras, contribuyendo a minimizar su impacto en el medio ambiente.

\section{REFERENCIAS}

Cerón, M. I., Santos, E. M., Sánchez, I., Rangel, E., Rodríguez, J. A., Ibarra, I. S. (2020). Hongos comestibles: Un ingrediente alternativo en la formulación de productos cárnicos. Publicación Semestral Pädi., 7 (14): 47-51. Recuperado de: https://www.researchgate.net/publication/338414316

Chockchaisa Wasdee, S., Namjaidee, S., Pochana, S., Stathopoulos, C. (2010). Development of fermented oyster - mushroom sausage. Asian Journal of 
Food and Agro-Industry. 3 (1): 35-43. Recuperado de: https://www.researchgate.net/publication/242242503_Development_of_f ermented_oyster-mushroom_sausage

De la Cruz, G. M., Morán, T., Huicab, Z. G., Rosales, V. (2020). El hongo Pleurotus ostreatus (Jacq. ex Fr.) y su valor agregado: caso de estudio. Revista Agroproductividad, 13(5): 73-78. Recuperado de: https://doi.org/10.32854/agrop.vi.1647

García, N., Bermúdez, R., Serrano, M. (2011). Formulaciones de sustratos en la producción de setas comestibles Pleurotus. Tecnología Química, 31(3), 1522. Universidad de Oriente, Santiago de Cuba, Cuba.

González, M. I. (2016) Alimentos funcionales obtenidos a partir de hongos nutraceúticos. Monografía en opción al título de Químico Industrial. Pereira, Colombia. Recuperado de: http://repositorio.utp.edu.co/dspace/handle/11059/6919

Heredia, A., Esparza, E. L., Romero, L., Cabral, F. J., Echavarría, F. G., Bañuelos. R. (2016). Evaluación de mezclas para sustrato y producción de Pleurotus ostreatus (jacq. ex fr.) p. Kumm. Revista Agroproductividad 9 (6): 67-72. Recuperado de: https://www.researchgate.net/publication/305905170

Hurtado, K., Huaman, M., Bravo, N., Silva, A., Silva, R. (2016). Evaluación del cultivo de Pleurotus ostreatus en mazorcas de cacao (Theobroma cacao L.). Rev. Per. Quím. Ing. Quím. 19 (1): 63-75. Recuperado de: https://www.researchgate.net/publication/354867705

Jaramillo, D., Yepes, L., Hincapié, G., Velásquez, A., Vélez, L. (2011). Desarrollo de productos a partir de la orellana (Pleurotus ostreatus). Revista Investigaciones Aplicadas 10: 32-41. Recuperado de: https://www.researchgate.net/publication/326468499

Magalhães, A. C., de A. B., R., Zied, D. C. (2018). Axenic cultivation of Pleurotus ostreatus var. Florida in supplemented sugarcane bagasse briquettes. Revista Engenharia Agrícola 38 (6): 835-843. Recuperado de: https://www.researchgate.net/publication/329693162

Piña, A. B., Nieto, D. A., Robles, F. (2016). Utilización de residuos agrícolas y agroindustriales en el cultivo y producción del hongo comestible seta (Pleurotus spp.). Rev. Int. Contam. Ambie., 32: 141-151. Recuperado de: https://www.researchgate.net/publication/312668895

Puig, Y., Crespo, L. M., Cardona, Y. R.; Matos, L., Serrano, M. (2020). Evaluación de tres residuos agroindustriales como sustratos para cultivo del Pleurotus Ostreatus var. Florida. Revista Científica Multidisciplinaria Arbitrada 
Yachasun., 4 (7): 164-176. Recuperado de: https://www.researchgate.net/publication/343929110

Ramírez, A. C. (2009) Estudio de las propiedades bioactivas de hongos comestibles para el diseño de productos cárnicos funcionales. Tesis en opción al grado científico de Doctor en Ciencias. Madrid, España. Recuperado de: https://repositorio.uam.es/handle/10486/117

Rangel, E., Rodríguez, J. A., Domínguez, R., Lorenzo, J. M., Sosa, M. E., Andres, S. C., Rosmini, M., Pérez-Álvarez, J. A., Teixeira, A., Santos, E. M. (2021). Edible Mushrooms as a Natural Source of Food Ingredient/Additive Replacer. Journal of Food., 10 (2687). Recuperado de: https://www.researchgate.net/publication/355886572

Rodríguez, G. E., Martínez, D. A., Buglione, M. B., Filippi, M. V., Agüero, M. S. (2018). Cultivo de Pleurotus ostreatus (Jacq.: Fr.) Kummer sobre orujo de pera: Evaluación de la productividad y composición química del sustrato biodegradado. Revista Anales de Biología 40: 21-30. Recuperado de: https://www.researchgate.net/publication/323701324

Ruilova, M. B., Hernández, A., Díaz, R., Niño-Ruiz, Z. (2016). Desarrollo de una salchicha saludable empleando al hongo Pleurotus ostreatus como sustituto de la carne de cerdo. Revista de Investigación Talentos, 3 (1): 36-41. Recuperado de https://talentos.ueb.edu.ec/index.php/talentos/article/view/67

Wan-Mohtar, W.A.A.Q.I., Halim-Lim, S.A., Kamarudin, N.Z., Rukayadi, Y., Abd Rahim, M.H., Jamaludin, A.A., Ilham, Z. (2020) Fruiting-body-base flour from an Oyster mushroom waste in the development of antioxidative chicken patty. Journal. Food Sci., 85 (10): 3124-3133. Recuperado de: https://doi.org/10.1111/1750-3841.15402

Wan Rosli, W., Solihah, M. (2012). Effect on the addition of Pleurotus sajor-caju (PSC) on physical and sensorial properties of beef patty. International Food Research Journal, 19 (3): 993-999. Recuperado de: https://www.researchgate.net/publication/288064825

Wan Rosli, W., Solihah, M., Mohsin, S. (2011). On the ability of oyster mushroom conferring changes in proximate composition andsensory evaluation of chicken patty. International Food Research Journal, 18 (4): 1463-1469. Recuperado de: https://www.researchgate.net/publication/286738858 